\title{
Modeling and controlling an active constrained layer (ACL) beam actuated by two voltage sources with/without magnetic effects
}

\author{
Ahmet Özkan Özer* \\ ${ }^{*}$ Department of Mathematics, Western Kentucky University, Bowling Green, KY 42101, USA
}

\begin{abstract}
A fully dynamic three-layer active constrained layer (ACL) beam is modeled for cantilevered boundary conditions by using a thorough variational approach. The Rao-Nakra thin compliant layer assumptions are adopted to model the sandwich structure, and all magnetic effects for the piezoelectric layers are retained. The piezoelectric layers are activated by two different voltage sources. When there are no "mechanical" boundary forces acting in the longitudinal direction, it is shown that the system with certain parameter combinations is not uniformly strongly stabilizable by the $B^{*}$-type feedback controller, which is the total current accumulated at the electrodes for the piezoelectric layers. However, as the magnetic effects are ignored (electrostatic assumption), the closedloop system with all mechanical feedback controllers is shown to be uniformly exponentially stable.
\end{abstract}

Index Terms - active constrained layer beam; smart sandwich beam, piezoelectric beam, Rao-Nakra beam, voltage controller, boundary feedback stabilization.

\section{INTRODUCTION}

An active constrained layer (ACL) composite beam consists of two piezoelectric layers and a constrained viscoelastic layer. Each piezoelectric layer is actuated by different voltage sources. When the electrodes of the piezoelectric layers are subjected to voltage sources, they shrink or extend, and therefore, the whole ACL composite shrinks/extends or bends. Accurately modeling the composite requires certain mechanical and electrical (and magnetic) assumptions for each layer. The middle layer is modeled by classical Mindlin-Timoshenko assumptions and the stiff piezoelectric layers are modeled by the Eulerbernoulli assumptions. Piezoelectric layers are traditionally modeled through the electrostatic assumption, and all dynamic electrical effects and magnetic effects due to Maxwell's equations are ruled out, i.e. see [19], and the references therein. Since an ACL composite includes a piezoelectric layer, the corresponding models use the electrostatic assumption as well [22]. The reduced model ([1], [7, [18]) is mostly either a Mead-Marcus-type [8] or a Rao-Nakra-type model [16. For example, [1] obtained a Mead-Marcus type model by neglecting the rotational inertia terms for the longitudinal dynamics and rotational inertia for the bending dynamics. All of these models reduce to the classical counterparts as in $([3],[5],[8])$ once the piezoelectric strain is taken to be zero. On the other hand, the model obtained in [18 through a consistent variational approach is more like a Rao-Nakra-type [16].

As the electrostatic assumption is adopted, it can be easily shown that a single piezoelectric beam model is exactly controllable and uniformly exponentially stabilizable for the $B^{*}$-type mechanical feedback, velocity of the beam at one end. The same type of phenomenon is observed for the ACL composite. For example in [1, it is shown that the time derivative of the energy is nonnegative as a mechanical damping is injected through the boundary of the piezoelectric layer. See other control strategies i.e. in [20, and the references therein. However, even in the case of a single piezoelectric beam, as the dynamic effects are kept, a strongly coupled wave system is obtained for which it is shown that the model is not controllable and not uniformly exponentially stabilizable for almost all combinations of material parameters with the $B^{*}$-type feedback, i.e. the total current at the electrodes [10]. In fact, there are no other feedback controllers to make system uniformly exponentially stable. It is worthwhile to mention that the closed-loop system obtained by supplying voltage to the electrodes and feeding back the total current is much easier and physical in terms of practical applications since measuring the total current at the electrodes is easier than measuring displacements or the velocity of the composite at one end of the beam, i.e. see ([2, [9]). In fact, with the same feedback controller, explicit polynomial decay estimates are obtained for more regular initial data 12. These results for a single piezoelectric beam indicate that a similar type of controllability characteristics may be observed for composites involving piezoelectric layers, i.e. the closed-loop system for an ACL composite with a similar type of input-output mentioned above may be more physical, and moreover, the ACL composite beam models with the magnetic effects may lack stabilizability/controllability for some choices of their parameters.

To our knowledge, magnetic effects are ignored for any smart composite involving a piezoelectric layer. In this paper, a novel modeling strategy is proposed to obtain models of an ACL composite with/without the magnetic effects by using the Rao-Nakra assumptions [16]. We further let the weight and the stiffness of the middle layer go to zero, i.e. see [4], to obtain reduced models which are 
the perturbations of the original models. Therefore, our models differ from the classical counterparts substantially not only due the inclusion of the magnetic effects but also due to the simplicity of the coupling between the longitudinal and bending dynamics, i.e. see ([1], [7]). A preliminary model has recently been obtained for an ACL composite with a single piezoelectric layer [13. In the modeling process, more attention is paid to the piezoelectric layers by using the full set of Maxwell's equations to incorporate the magnetic effects. We prove the lack of uniform strong stabilization with the $B^{*}$-type feedback in the case where the mechanical boundary forces for the longitudinal dynamics are removed. Next, we consider the model without magnetic effects. This model is reduced to the "nonsmart" Rao-Nakra composite [4]. To our knowledge, the boundary feedback stabilization or exact controllability for the clamped-free Rao-Nakra beam model were never considered in the literature, i.e. see 15 and the references therein, except the result for the clamped-hinged boundary conditions where each equation for stretching and bending requires only one controller. In this paper, our results require two controllers for the bending equation. By adopting a similar argument of [15, it is shown that the reduced model is a compact perturbation of the decoupled system consisting of a Rayleigh (Kirchhoff) beam equation [17] and wave equations. Therefore the coupled system is shown to be exponentially stable since the unique continuation result proves that there are no eigenvalues on the imaginary axis, see Lemma 4.2 Unlike the clampedhinged ACL beam [15, our result requires using the four boundary conditions at one end which means that two controllers are essential to control the bending motion for the smart beam.

\section{Modeling Preliminaries}

Consider an ACL beam occupying the region $\Omega=$ $\Omega_{x y} \times(0, h)=[0, L] \times[-b, b] \times(0, h)$ at equilibrium where $\Omega_{x y}$ is a smooth bounded domain in the plane. The total thickness $h$ is assumed to be small in comparison to the dimensions of $\Omega_{x y}$. The beam consists of two piezoelectric layers and a complaint layer. The layers are indexed from 1 to 3 from the bottom piezoelectric layer to the top piezoelectric layer, respectively. Now we let $0=z_{0}<$ $z_{1}<z_{2}<z_{3}=h$, with $h_{i}=z_{i}-z_{i-1}, \quad i=1,2,3$. We use the rectangular coordinates $(x, y)$ to denote points in $\Omega_{x y}$, and $(X, z)$ to denote points in $\Omega=\Omega_{\mathrm{B}} \cup \Omega^{\mathrm{ve}} \cup \Omega^{\mathrm{T}}$, where $\Omega_{\mathrm{B}}=\Omega_{x y} \times\left(z_{0}, z_{1}\right), \Omega^{\mathrm{ve}}=\Omega_{x y} \times\left(z_{1}, z_{2}\right)$, and $\Omega^{\mathrm{T}}=\Omega_{x y} \times\left(z_{2}, z_{3}\right)$ are the reference configurations of the bottom piezoelectric, viscoelastic, and top piezoelectric layers, respectively.

For $(x, y, z) \in \Omega$, let $U(x, y, z)=\left(U_{1}, U_{2}, U_{3}\right)(x, y, z)$ denote the displacement vector of the point (from reference configuration). In order to obtain a beam theory, all displacements are assumed to be independent of $y$-coordinate, and $U_{2} \equiv 0$. The transverse displacements is $w(x, y, z)=U_{3}(x)=w^{i}(x)$ for any $i$ and $x \in[0, L]$. Define $u^{i}(x, y, z)=U_{1}\left(x, 0, z_{i}\right)=u^{i}(x)$ for $i=0,1,2,3$ and for all $x \in(0, L)$.
We use the standard sandwich beam assumptions to model the ACL beams. The modified constitutive equations for the piezoelectric layers are

$$
\left\{\begin{array}{l}
T_{11}^{i}=\alpha^{i} S_{11}^{(i)}-\gamma^{i} \beta^{i} D_{3}^{i}, \quad E_{1}^{i}=\beta_{1}^{i} D_{1}^{i} \\
E_{3}^{i}=-\gamma^{i} \beta^{i} S_{11}^{i}+\beta^{i} D_{3}^{i}, \quad i=1,3
\end{array}\right.
$$

where $T, S, D, E, c_{11}, \gamma, \epsilon$, and $\beta$; are stress tensor, strain tensor, electrical displacement vector, electric field intensity vector, elastic stiffness coefficients, piezoelectric coefficients, permittivity coefficients, impermittivity coefficients, and $\alpha^{i}=\alpha_{1}^{i}+\left(\gamma^{i}\right)^{2} \beta^{i}, \quad \alpha_{1}^{i}=c_{11}^{i}, \quad \alpha^{2}=c_{11}^{2}$ and $\gamma^{i}=\gamma_{31}^{i}, \quad \gamma_{1}^{i}=\gamma_{15}^{i}, \quad \beta^{i}=\frac{1}{\varepsilon_{33}^{i}}, \quad \beta_{1}^{i}=\frac{1}{\varepsilon_{11}^{i}}$, and the middle layer is $T_{11}=\alpha_{1}^{2} S_{11}, \quad T_{13}=2 G_{2} S_{13}$ where $G_{2}$ is the shear modulus of the viscoelastic layer, and refer to ([13, [19) for the description of piezoelectric and elasticity coefficients. Defining $\hat{z}_{i}=\frac{z^{i-1}+z^{i}}{2}$, the strain components for the viscoelastic layer, and the piezoelectric layers are respectively given by

$$
\begin{gathered}
S_{11}=\frac{\partial v^{2}}{\partial x}-\left(z-\hat{z}_{i}\right) \frac{\partial \psi^{2}}{\partial x}, \quad S_{13}=\frac{1}{2}\left(\psi^{2}+w_{x}\right)=\frac{1}{2} \phi^{2} \\
S_{11}^{i}=\frac{\partial v^{i}}{\partial x}-\left(z-\hat{z}_{i}\right) \frac{\partial^{2} w}{\partial x^{2}}, \quad S_{13}^{i}=0, \quad i=1,3, \\
\psi^{i}=\frac{u^{i}-u^{i-1}}{h_{i}}, \quad \phi^{i}=\psi^{i}+w_{x}, \quad v^{i}=\frac{u^{i-1}+u^{i}}{2}
\end{gathered}
$$

where $i=1,2,3$, and in particular, $\phi^{1}=\phi^{3}=0, \quad \psi^{1}=$ $\psi^{3}=-w_{x}, \quad \phi^{2}=\psi^{2}+w_{x}$. Here $\psi^{i}$ can be viewed as the total rotation angles of the deformed filament within the $i^{\text {th }}$ layer in the $x-z$ plane, and $\phi^{i}$ represent the shear angles within each layer, and $v^{i}$ represent the longitudinal displacements of the center line of the $i^{\text {th }}$ layer. For details of the constitutive equations and parameters, the reader may refer to $([4],[10]$, or [13]).

Inclusion of the electrical kinetic energy for the piezoelectric layers: Let $\mathrm{B}^{\mathrm{i}}$ be the magnetic field $\mathrm{B}^{i}(x)$ for the $i^{\text {th }}$ piezoelectric layer for $i=1,3$, and have the only nonzero component $\mathrm{B}_{2}^{i}(x)$. Assume also that the electric field of the $i^{\text {th }}$ piezoelectric layer $E_{1}^{i}=0$, and thus $D_{1}^{i}=0$. Assuming that $D_{3}^{i}$ does not vary in the thickness direction $D_{3}^{i}(x, z, t)=D_{3}^{i}(x, t)$, it follows from the AmpéreMaxwell equation that $\mathrm{B}_{2}^{i}=-\mu^{i} \int_{0}^{x} \dot{D}_{3}^{i}(\xi, z, t) d \xi$ where $\mu^{i}$ is the magnetic permeability of the $i^{\text {th }}$ layer. Now we define $p^{i}:=\int_{0}^{x} D_{3}^{i}(\xi, t) d \xi$ to be the total electric charge at point $x$. The magnetic energy for the $i^{\text {th }}$ layer is $B^{i}=\frac{\mu^{i}}{2} \int_{\Omega}\left(\dot{p}^{i}\right)^{2} d X$.

Assume that the beam is subject to a distribution of boundary forces $\left(\tilde{g}^{1}, \tilde{g}^{3}, \tilde{g}\right)$ along its edge $x=L$, see [6]. Let $V^{\mathrm{T}}(t)$ and $V_{\mathrm{B}}(t)$ be the voltages applied at the electrodes of the piezoelectric layers, respectively. Then the total work done by all mechanical and electrical external forces is

$$
\begin{aligned}
\mathbf{W}= & \int_{0}^{L}\left(-\left(p^{1}\right)_{x} V_{\mathrm{B}}-\left(p^{3}\right)_{x} V^{\mathrm{T}}\right) d x+g^{1} v^{1}(L) \\
& +g^{3} v^{3}(L)+g w(L)-M w_{x}(L)
\end{aligned}
$$

where $M=m^{1}+m^{3}, g^{i}=\int_{z_{i-1}}^{z_{i}} \tilde{g}^{i} d z, g=\int_{0}^{h} \tilde{g} d z, m^{i}=$ $\int_{z_{i-1}}^{z_{i}}\left(z-\hat{z}^{i}\right) \tilde{g}_{i} d z$ for $i=1,3$. The modified Lagrangian for the ACL beam is $\mathbf{L}=\int_{0}^{T}[\mathbf{K}-(\mathbf{P}+\mathbf{E})+\mathbf{B}+\mathbf{W}] d t$ where $\mathbf{K}=\sum_{i=1}^{3} \mathbf{K}^{i}, \mathbf{P}+\mathbf{E}=\mathbf{P}^{2}+\sum_{i=1,3}\left(\mathbf{P}^{i}+\mathbf{E}^{i}\right)$, 
and $\mathbf{B}=\mathbf{B}^{1}+\mathbf{B}^{3}$ are the kinetic energy, the total stored energy, and the magnetic energy of the beam [10],

$$
\begin{aligned}
& \mathbf{K}=\frac{1}{2} \int_{0}^{L}\left[\left(\sum_{i=1,3} \rho_{i} h_{i}\left(\dot{v}^{i}\right)^{2}\right)+\left(\sum_{i=1,3} \rho_{i} h_{i}\right) \dot{w}^{2}\right. \\
& \left.\quad+\rho_{2} h_{2}\left(\dot{\psi}^{2}\right)^{2}+\left(\rho_{1} h_{1}+\rho_{3} h_{3}\right) \dot{w}_{x}^{2}\right] d x, \\
& \mathbf{P}+\mathbf{E}=\frac{1}{2} \int_{0}^{L}\left[\alpha^{2} h_{2}\left(\left(v_{x}^{2}\right)^{2}+\frac{h_{2}^{2}}{12}\left(\psi_{x}\right)^{2}\right)\right. \\
& +G_{2} h_{2}\left(\phi^{2}\right)^{2}+\sum_{i=1,3}\left(\alpha^{i} h_{i}\left(\left(v_{x}^{i}\right)^{2}+\frac{h_{i}^{2}}{12}\left(w_{x x}\right)^{2}\right)\right. \\
& \left.\left.-2 \gamma^{i} \beta^{i} h_{i} v_{x}^{i} p_{x}^{i}+\beta^{i} h_{i}\left(p_{x}^{i}\right)^{2}\right)\right] d x, \\
& \mathbf{B}=\frac{1}{2} \int_{0}^{L} \sum_{i=1,3}\left(\mu^{i} h_{i}\left(\dot{p}^{i}\right)^{2}\right) d x
\end{aligned}
$$

where $\rho_{i}$ is the volume density of the $i{ }^{\text {th }}$ layer. Refer to (4], [10]) for the details.

\section{RAO-NAKRA MOdel AND HAMILTON'S PRINCIPLE}

By using (3), $\left\{v^{2}, \psi^{2}\right\}$ can be written as functions of $\left\{w, v^{1}, v^{3}\right\}$. Thus, we choose only $\left\{w, v^{1}, v^{3}\right\}$ as the state variables. Let $H=\frac{h_{1}+2 h_{2}+h_{3}}{2}$. Application of Hamilton's principle by using cantilevered boundary conditions and by setting the variation of admissible displacements $\left\{v^{1}, v^{3}, p^{1}, p^{3}, w\right\}$ of $\mathbf{L}$ to zero yields a highly coupled equations for bending and stretching of the whole composite. Thus, we study the thin compliant layer Rao-Nakra model by letting $\rho_{2}, \alpha^{2} \rightarrow 0$ :

$$
\left\{\begin{array}{l}
\rho_{i} h_{i} \ddot{v}^{i}-\alpha^{i} h_{i} v_{x x}^{i}+\gamma^{i} \beta^{i} h_{i} p_{x x}^{i}+\kappa(i) G_{2} \phi^{2}=0, \\
\mu^{i} h_{i} \ddot{p}^{i}-\beta^{i} h_{i} p_{x x}^{i}+\gamma^{i} \beta^{i} h_{i} v_{x x}^{i}=0, \quad i=1,3, \\
m \ddot{w}-K_{1} \ddot{w}_{x x}+K_{2} w_{x x x x}-G_{2} H \phi_{x}^{2}=0, \\
\phi^{2}=\frac{1}{h_{2}}\left(-v^{1}+v^{3}+H w_{x}\right)
\end{array}\right.
$$

with the boundary and initial conditions for $i=1,3$

$$
\left\{\begin{array}{l}
v^{i}(0)=p^{i}(0), \alpha^{i} h_{i} v_{x}^{i}(L)-\gamma^{i} \beta^{i} h_{i} p_{x}^{i}(L)=g^{i}(t), \\
\beta^{1} h_{1} p_{x}^{1}(L)-\gamma^{1} \beta^{1} h_{1} v_{x}^{1}(L)=-V_{\mathrm{B}}(t) \\
\beta^{3} h_{3} p_{x}^{3}(L)-\gamma^{3} \beta^{3} h_{3} v_{x}^{3}(L)=-V^{\mathrm{T}}(t) \\
w(0)=w_{x}(0)=0, \quad K_{2} w_{x x}(L)=-M(t) \\
K_{1} \ddot{w}_{x}(L)-K_{2} w_{x x x}(L)+G_{2} H \phi^{2}(L)=g(t) \\
\left(v^{1}, v^{3}, p^{1}, p^{3}, w, \dot{v}^{1}, \dot{v}^{3}, \dot{p}^{1}, \dot{p}^{3}, \dot{w}\right)(x, 0) \\
\quad=\left(v_{0}^{1}, v_{0}^{3}, p_{0}^{1}, p_{0}^{3}, w_{0}, v_{1}^{1}, v_{1}^{3}, p_{1}^{1}, p_{1}^{3}, w_{1}\right)
\end{array}\right.
$$

where $\kappa(i)=\operatorname{sgn}(i-2), m=\sum_{i=1}^{3} \rho_{i} h_{i}, K_{1}=\frac{\rho_{1} h_{1}^{3}}{12}+\frac{\rho_{3} h_{3}^{3}}{12}$, and $K_{2}=\frac{\alpha^{1} h_{1}^{3}}{12}+\frac{\alpha^{3} h_{3}^{3}}{12}$. Note that, different from a single piezoelectric model, the voltage controls $V^{\mathrm{T}}(t)$ and $V_{\mathrm{B}}(t)$ strongly couple the stretching and bending equations due the shear effect $\phi^{2}$ of the middle layer.

Note that the role of the longitudinally applied mechanical boundary feedback controllers $g^{1}$ and $g^{3}$ are crucial to obtain a uniform exponential stabilization result (one control for each equation). However, once the mechanical boundary controllers for the stretching equations are removed, i.e. $g^{1}, g^{3} \equiv 0$, the system is not even strongly stable by the $B^{*}$-type feedback for certain choices of material parameters. For $i=1,3$ define

$$
\begin{array}{r}
\zeta_{ \pm}^{i}=\frac{\sqrt{\frac{\left(\gamma^{i}\right)^{2} \mu^{i}}{\alpha_{1}^{i}}+\frac{\mu^{i}}{\beta^{i}}+\frac{\rho_{i}}{\alpha_{1}^{i}} \pm \sqrt{\left(\frac{\left(\gamma^{i}\right)^{2} \mu^{i}}{\alpha_{1}^{i}}+\frac{\mu^{i}}{\beta^{i}}+\frac{\rho^{i}}{\alpha_{1}^{i}}\right)^{2}-\frac{4 \rho^{i} \mu^{i}}{\beta^{i} \alpha_{1}^{i}}}}}{\sqrt{2}} \\
b_{ \pm}^{i}=\frac{1}{2}\left(\gamma^{i}+\frac{\alpha_{1}^{i}}{\gamma^{i} \beta^{i}}-\frac{\rho_{i}}{\gamma^{i} \mu^{i}} \pm \sqrt{\left(\gamma^{i}+\frac{\alpha_{i}^{1}}{\gamma^{i} \beta^{i}}-\frac{\rho_{i}}{\gamma^{i} \mu^{i}}\right)^{2}+\frac{4 \rho_{i}}{\mu^{i}}}\right)
\end{array}
$$

where $\zeta_{+}, \zeta_{-}, b_{-}, b_{+} \neq 0, b_{-} \neq b_{+}, \zeta_{+} \neq \zeta_{-}$with $\zeta_{+} \zeta_{-}=$ $\sqrt{\frac{\rho_{i} \mu^{i}}{\beta^{i} \alpha_{1}^{i}}}, b_{-} b_{+}=\frac{\rho_{i}}{\mu_{i}}$. Let two piezoelectric beams have the same material properties, i.e. $\alpha_{1}^{1}=\alpha_{1}^{3}, \gamma^{1}=\gamma^{3}$, etc. Under no mechanical longitudinal forces $g^{1}=g^{3} \equiv 0$, we have the following result:

Theorem 3.1: The system (6)-(7) with $g^{1}, g^{3} \equiv 0$ is not strongly stable by the feedback, i.e. $V^{\mathrm{T}}(t)=\frac{s_{3} \dot{p}^{3}(L)}{2 h_{3}}$, $V_{\mathrm{B}}(t)=\frac{s_{1} \dot{p}^{1}(L)}{2 h_{1}}, M(t)=-k_{1} \dot{w}_{x}(L)$ and $g(t)=k_{2} \dot{w}(L)$ for $s_{1}, s_{2}, k_{1}, k_{2}>0$ if $\frac{\zeta_{+}^{i}}{\zeta_{-}^{i}}=\frac{2 n_{i}-1}{2 m_{i}-1}$ for some $m_{i}, n_{i} \in \mathbb{N}, i=$ 1,3 .

Proof: We prove that there are eigenvalues on the imaginary axis. Consider the eigenvalue problem corresponding to (6)-(7) with $\lambda=\imath \tau$ :

$$
\left\{\begin{array}{l}
\alpha_{1}^{i} h_{i} v_{x x}^{i}-\gamma^{i} \beta^{i} h_{i} p_{x x}^{i}-\kappa(i) G_{2} \phi^{2}=-\tau^{2} \rho_{i} h_{i} z^{i} \\
\beta^{i} h_{i} p_{x x}^{i}-\gamma^{i} \beta^{i} h_{i} v_{x x}^{i}=-\tau^{2} \mu^{i} h_{i} p^{i}, \quad i=1,3 \\
-K_{2} w_{x x x x}+G_{2} H \phi_{x}^{2}=-\tau^{2}\left(m w-K_{1} w_{x x}\right)
\end{array}\right.
$$

with the overdetermined boundary conditions

$$
\begin{array}{r}
\left|w=w_{x}=v^{i}=p^{i}\right|_{x=0}=\left|v_{x}^{i}=p_{x}^{i}=p^{i}\right|_{x=L}=0, \\
w(L)=w_{x}(L)=w_{x x}(L)=w_{x x x}(L)=0, \quad i=1,3 .
\end{array}
$$

Let $w(x) \equiv 0$ and

$$
\begin{aligned}
v^{i}(x)= & k_{1}^{i} \frac{a_{+}^{i} b_{+}^{i} \sin \left(a_{-}^{i} x\right)-a_{-} b_{-}^{i} \sin \left(a_{+}^{i} x\right)}{a_{+}^{i} a_{-}^{i}\left(b_{+}^{i}-b_{-}^{i}\right)} \\
& +k_{2}^{i} \frac{-a_{+}^{i} \sin \left(a_{-}^{i} x\right)+a_{-}^{i} \sin \left(a_{+}^{i} x\right)}{a_{+}^{i} a_{-}^{i}\left(b_{+}^{i}-b_{-}^{i}\right)}, \\
p^{i}(x)= & k_{1}^{i} \frac{\left(a_{+}^{i} \sin \left(a_{-}^{i} x\right)-a_{-}^{i} \sin \left(a_{+}^{i} x\right)\right) b_{1} b_{2}}{a_{+}^{i} a_{-}^{i}\left(b_{+}^{i}-b_{-}^{i}\right)} \\
& +k_{2}^{i} \frac{a_{-}^{i} b_{+}^{i} \sin \left(a_{+}^{i} x\right)-a_{+}^{i} b_{-}^{i} \sin \left(a_{-}^{i} x\right)}{a_{+}^{i} a_{-}^{i}\left(b_{+}^{i}-b_{-}^{i}\right)}
\end{aligned}
$$

where $a_{+}^{i}=\tau \zeta_{+}^{i}=\frac{\left(2 n_{i}-1\right) \pi}{2 L}, a_{-}^{i}=\tau \zeta_{-}^{i}=$ $\frac{\left(2 m_{i}-1\right) \pi}{2 L}$ for some $m_{i}, n_{i} \in \mathbb{N}, i=1,3$, $k_{1}^{i}=\frac{a_{-}^{i} b_{+}^{i} \sin \left(a_{+}^{i} L\right)-a_{+}^{i} b_{-}^{i} \sin \left(a_{-}^{i} L\right)}{a_{+}^{i} a_{-}^{i}\left(b_{+}^{i}-b_{-}^{i}\right)}$, and $k_{2}^{i}=$ $-\frac{\left(a_{+}^{i} \sin \left(a_{-}^{i} L\right)-a_{-}^{i} \sin \left(a_{+}^{i} L\right)\right) b_{+}^{i} b_{-}^{i}}{a_{+}^{i} a_{-}^{i}\left(b_{+}^{i}-b_{-}^{i}\right)}$. Here $v^{1}=v^{3}, p^{1}=p^{3}, w \equiv$ $0, \phi^{2} \equiv 0$, and $\left(v^{1}, v^{3}, p^{1}, p^{3}, w\right)$ is the non-trivial solution of eigenvalue problem (8)-(9). This implies that there are eigenvalues on the imaginary axis; $\left\{ \pm \frac{\imath a_{+}^{i}}{\zeta_{+}^{i}}, \pm \frac{\imath a_{-}^{i}}{\zeta_{-}^{i}}\right\}$. The conclusion follows.

\section{Stabilization without magnetic efFects}

First, assume that the magnetic energy for each layer is zero, i.e. $\mathbf{B}^{i}=0$, and so, $\ddot{p}^{1}=\ddot{p}^{3} \equiv 0$ in 10 . The 
electrostatic model is the well-known Rao-Nakra model in 4. The boundary stabilization problem is well studied in [15] for the multi-layer beam clamped at the left end and hinged at the right end.

Finally, for $k_{1}, k_{2}, s_{1}, s_{3}>0$, analogous to [6] and [17], we consider the following system

$$
\left\{\begin{array}{l}
\rho_{i} h_{i} \ddot{v}^{i}-\alpha_{1}^{i} h_{i} v_{x x}^{i}+\kappa(i) G_{2} \phi^{2}=0, \quad i=1,3 \\
m \ddot{w}-K_{1} \ddot{w}_{x x}+K_{2} w_{x x x x}-G_{2} H \phi_{x}^{2}=0, \\
\phi^{2}=\frac{1}{h_{2}}\left(-v^{1}+v^{3}+H w_{x}\right)
\end{array}\right.
$$

with the boundary and initial conditions

$$
\begin{gathered}
v^{i}(0)=w(0)=w_{x}(0)=0, \quad K_{2} w_{x x}(L)=-k_{1} \dot{w}_{x}(L) \\
\alpha_{1}^{i} h_{i} v_{x}^{i}(L)=-s_{i} \gamma^{i} \dot{v}^{i}(L), \quad i=1,3 \\
K_{1} \ddot{w}_{x}(L)-K_{2} w_{x x x}(L)+G_{2} H \phi^{2}(L)=k_{2} \dot{w}(L) \\
\left(v^{1}, v^{3}, w, \dot{v}^{1}, \dot{v}^{3}, \dot{w}\right)(x, 0)=\left(v_{0}^{1}, v_{0}^{3}, w_{0}, v_{1}^{1}, v_{1}^{3}, w_{1}\right) .(11)
\end{gathered}
$$

Semigroup well-posedness: Define $H_{L}^{1}(0, L)=\{\psi \in$ $\left.H^{1}(0, L): \psi(0)=0\right\}, H_{L}^{2}(0, L)=\left\{\psi \in H^{2}(0, L): \psi(0)=\right.$ $\left.\psi_{x}(0)=0\right\}$, and the complex linear spaces

$$
\begin{aligned}
& \mathbb{X}=\mathbb{L}^{2}(0, L), \quad \mathrm{V}=\left(H_{L}^{1}(0, L)\right)^{2} \times H_{L}^{2}(0, L), \\
& \mathrm{H}=\mathbb{X}^{2} \times H_{L}^{1}(0, L), \quad \mathcal{H}=\mathrm{V} \times \mathrm{H} .
\end{aligned}
$$

so that $\mathrm{V} \subset \mathrm{H} \subset \mathbb{X}^{3} \subset \mathrm{H}^{\prime} \subset \mathrm{V}^{\prime}$. The natural energy associated with $10-(11)$ is

$$
\begin{aligned}
& \mathrm{E}(t)=\frac{1}{2} \int_{0}^{L}\left\{\sum_{i=1,3}\left(\rho_{i} h_{i}\left|\dot{v}^{i}\right|^{2}+\alpha_{1}^{i} h_{i}\left|v_{x}^{i}\right|^{2}\right)+m|\dot{w}|^{2}\right. \\
& \left.+K_{1}\left|\dot{w}_{x}\right|^{2}+K_{2}\left|w_{x x}\right|^{2}+G_{2} h_{2}\left|\phi^{2}\right|^{2}\right\} d x
\end{aligned}
$$

This motivates definition of the inner product on $\mathcal{H}$

$$
\begin{aligned}
& \left\langle\left[\begin{array}{c}
u_{1} \\
\vdots \\
u_{6}
\end{array}\right],\left[\begin{array}{l}
v_{1} \\
\vdots \\
v_{6}
\end{array}\right]\right\rangle_{\mathcal{H}}=\left\langle\left[\begin{array}{l}
u_{4} \\
u_{5} \\
u_{6}
\end{array}\right],\left[\begin{array}{l}
v_{4} \\
v_{5} \\
v_{6}
\end{array}\right]\right\rangle_{\mathrm{H}} \\
& \quad+\left\langle\left[\begin{array}{c}
u_{1} \\
u_{2} \\
u_{3}
\end{array}\right],\left[\begin{array}{c}
v_{1} \\
v_{2} \\
v_{3}
\end{array}\right]\right\rangle_{\mathrm{V}} \\
& =\int_{0}^{L}\left\{\rho_{1} h_{1} u_{4} \dot{\bar{v}}_{4}+\rho_{3} h_{3} u_{5} \dot{\bar{v}}_{5}+m \dot{u}_{6} \dot{\bar{v}}_{6}+K_{1}\left(u_{6}\right)_{x}\left(\bar{v}_{6}\right)_{x}\right. \\
& +\alpha_{1}^{1} h_{1}\left(u_{1}\right)_{x}\left(\bar{v}_{1}\right)_{x}+\alpha_{1}^{3} h_{3}\left(u_{2}\right)_{x}\left(\bar{v}_{2}\right)_{x}+K_{2}\left(u_{3}\right)_{x x}\left(\bar{v}_{3}\right)_{x x} \\
& \left.+\frac{G_{2}}{h_{2}}\left(-u_{1}+u_{2}+H\left(u_{3}\right)_{x}\right)\left(-\bar{v}_{1}+\bar{v}_{2}+H\left(\bar{v}_{3}\right)_{x}\right)\right\} d x .
\end{aligned}
$$

Obviously, $\langle,\rangle_{\mathcal{H}}$ does indeed define an inner product, with the induced energy norm since the term $\|-u_{1}+u_{2}+$ $H\left(u_{4}\right)_{x} \|_{L^{2}(0, L)}$ is coercive, see [4] for the details.

Let $\vec{y}=\left(v^{1}, v^{3}, w\right)$ be the smooth solution of the system of (10)-(11). Assuming the homogenous problem, all external forces are zero, multiplying the equations in (10) by $\tilde{y}_{1}, \tilde{y}_{3}, \in H_{L}^{1}(0, L)$ and $\tilde{y} \in H_{L}^{2}(0, L)$, respectively, and integrating by parts yields

$$
\begin{aligned}
& \begin{array}{l}
\int_{0}^{L}\left(\rho_{i} h_{i} \ddot{v}^{i} \tilde{y}_{i}+\alpha_{1}^{i} h_{i} v_{x}^{i}\left(\tilde{y}_{i}\right)_{x}+\kappa(i) G_{2} \phi^{2} \tilde{y}_{i}\right) d x \\
\quad+s_{i} \dot{v}^{i}(L) \tilde{y}_{i}(L)=0, \quad i=1,3, \\
\int_{0}^{L}\left(m \ddot{w} \tilde{y}+K_{1} \ddot{w}_{x} \tilde{y}_{x}+K_{2} w_{x x} \tilde{y}_{x x}-G_{2} H \phi_{x}^{2} \tilde{y}_{x}\right) d x \\
+k_{1} \dot{w}_{x}(L) \tilde{y}_{x}(L)+k_{2} \dot{w}(L) \tilde{y}(L)=0 .
\end{array}
\end{aligned}
$$

Now define the linear operators

$$
\begin{array}{r}
\langle A y, \psi\rangle_{\mathrm{V}^{\prime} \times \mathrm{V}}=(y, \psi)_{\mathrm{V} \times \mathrm{V}}, \forall y, \psi \in \mathrm{V} \\
\left\langle B_{0} \vec{y}, \vec{\psi}\right\rangle_{\mathrm{H}^{\prime} \times \mathrm{H}}=\left[\begin{array}{c}
0_{2 \times 1} \\
k_{2} y_{3}(L) \psi_{3}(L)
\end{array}\right], \forall \vec{y}, \vec{\psi} \in \mathrm{H} \\
\left\langle D_{0} \vec{y}, \vec{\psi}\right\rangle_{\mathrm{H}^{\prime} \times \mathrm{H}}=\left[\begin{array}{c}
s_{1} y_{1}(L) \psi_{1}(L) \\
s_{3} y_{2}(L) \psi_{2}(L) \\
k_{1}\left(y_{3}\right)_{x}(L)\left(\psi_{3}\right)_{x}(L)
\end{array}\right], \forall \vec{y}, \vec{\psi} \in \mathrm{V} .
\end{array}
$$

Let $\mathcal{M}: H_{L}^{1}(0, L) \rightarrow\left(H_{L}^{1}(0, L)\right)^{\prime}$ be a linear operator defined by

$$
\langle\mathcal{M} \psi, \tilde{\psi}\rangle_{\left(H_{L}^{1}(0, L)\right)^{\prime}, H_{L}^{1}(0, L)}=\int_{0}^{L}\left(m \psi \tilde{\bar{\psi}}+K_{1} \psi_{x} \tilde{\bar{\psi}}_{x}\right) d x .
$$

From the Lax-Milgram theorem $\mathcal{M}$ and $A$ are canonical isomorphisms from $H_{L}^{1}(0, L)$ onto $\left(H_{L}^{1}(0, L)\right)^{\prime}$ and from $V$ onto $V^{\prime}$, respectively. Assume that $A y \in V^{\prime}$, then we can formulate the variational equation above into the following form $M \ddot{y}+A y+D_{0} \dot{y}+B_{0} \dot{y}=0$ where $M=\left[\begin{array}{lll}\rho_{1} h_{1} I & \rho_{3} h_{3} I & \mathcal{M}\end{array}\right]$ is an isomorphism from $\mathrm{H}$ onto $\mathrm{H}^{\prime}$. Next we introduce the linear unbounded operator by

$$
\mathcal{A}: \operatorname{Dom}(A) \times V \subset \mathcal{H} \rightarrow \mathcal{H}
$$

where $\mathcal{A}=\left[\begin{array}{cc}O_{3 \times 3} & -I_{3 \times 3} \\ M^{-1} A & M^{-1} D_{0}\end{array}\right]$ with $\operatorname{Dom}(\mathcal{A})=$ $\left\{(\vec{z}, \overrightarrow{\tilde{z}}) \in V \times V, A \vec{z} \in \mathrm{V}^{\prime}\right\}$, and if $\operatorname{Dom}(\mathcal{A})^{\prime}$ is the dual of $\operatorname{Dom}(\mathcal{A})$ pivoted with respect to $\mathcal{H}$, we define the control operator $B$

$$
B \in \mathcal{L}\left(\mathbb{C}, \operatorname{Dom}(\mathcal{A})^{\prime}\right), \text { with } B=\left[\begin{array}{c}
0_{3 \times 1} \\
M^{-1} B_{0}
\end{array}\right]
$$

Writing $\varphi=\left[v^{1}, v^{3}, w, \dot{v}^{1}, \dot{v}^{3}, \dot{w}\right]^{\mathrm{T}}$, the control system 10)-11 with the feedback controllers can be put into the state-space form

$$
\dot{\varphi}+\mathcal{A} \varphi+B \varphi=0, \quad \varphi(x, 0)=\varphi^{0} .
$$

Lemma 4.1: The operator $\mathcal{A}$ defined by 16 is maximal monotone in the energy space $\mathcal{H}$, and Range $(I+\mathcal{A})=\mathcal{H}$.

Proof: Let $\vec{z}=\left[\begin{array}{c}\vec{z}_{1} \\ \vec{z}_{2}\end{array}\right] \in \operatorname{Dom}(\mathcal{A})$. A simple calculation using integration by parts and the boundary conditions yields

$$
\begin{aligned}
\langle\mathcal{A} \vec{z}, & \vec{z}\rangle_{\mathcal{H} \times \mathcal{H}}=\left\langle\left[\begin{array}{c}
-\vec{z}_{2} \\
M^{-1}\left(A \vec{z}_{1}+D_{0} \vec{z}_{2}\right)
\end{array}\right],\left[\begin{array}{c}
\vec{z}_{1} \\
\vec{z}_{2}
\end{array}\right]\right\rangle_{\mathcal{H}} \\
& =\left\langle-\vec{z}_{2}, \vec{z}_{1}\right\rangle_{V \times V}+\left\langle M^{-1}\left(A \vec{z}_{1}+D_{0} \vec{z}_{2}\right), \vec{z}_{2}\right\rangle_{H \times H} \\
& =-\overrightarrow{\left\langle A \vec{z}_{1}, \vec{z}_{2}\right\rangle_{V^{\prime} \times V}}+\left\langle A \vec{z}_{1}+D_{0} \vec{z}_{2}, \vec{z}_{2}\right\rangle_{H^{\prime} \times H} .
\end{aligned}
$$

Since $\vec{z} \in \operatorname{Dom}(\mathcal{A})$, then $A \vec{z}_{1}+D_{0} \vec{z}_{2} \in V^{\prime}$ and $\vec{z}_{2} \in V$ so that

$$
\begin{array}{r}
\left\langle A \vec{z}_{1}+D_{0} \vec{z}_{2}, \vec{z}_{2}\right\rangle_{H^{\prime} \times H}=\left\langle A \vec{z}_{1}+D_{0} \vec{z}_{2}, \vec{z}_{2}\right\rangle_{V^{\prime} \times V} \\
=\left\langle A \vec{z}_{1}, \vec{z}_{2}\right\rangle_{V^{\prime} \times V}+\left\langle D_{0} z_{2}, \vec{z}_{2}\right\rangle_{V^{\prime} \times V}
\end{array}
$$

Hence plugging 20 in 190 yields $\operatorname{Re}\langle\mathcal{A} \vec{z}, \vec{z}\rangle_{\mathcal{H} \times \mathcal{H}}=$ $\left\langle D_{0} \vec{z}_{2}, \vec{z}_{2}\right\rangle_{V^{\prime} \times V} \geq 0$ by 14 . We next verify the range condition. Let $\vec{z}=\left[\begin{array}{c}\vec{z}_{1} \\ \vec{z}_{2}\end{array}\right] \in \mathcal{H}$. We prove that there exists a $\vec{y}=\left[\begin{array}{l}\vec{y}_{1} \\ \vec{y}_{2}\end{array}\right] \in \operatorname{Dom}(\mathcal{A})$ such that $(I+\mathcal{A}) \vec{y}=\vec{z}$. A 
simple computation shows that proving this is equivalent to proving Range $\left(M+A+D_{0}\right)=H^{\prime}$, i.e., for every $\vec{f} \in H^{\prime}$ there exists a unique solution $\vec{z} \in H$ such that $\left(M+A+D_{0}\right) \vec{z}=\vec{f}$. This obviously follows from the Lax Milgram's theorem.

Proposition 4.1: The operator $B$ is a monotone compact operator on $\mathrm{H}$.

Proof: Let $\left[\begin{array}{l}\vec{y} \\ \vec{z}\end{array}\right] \in \mathrm{H}$. Then $\left\langle B\left[\begin{array}{l}\vec{y} \\ \vec{z}\end{array}\right],\left[\begin{array}{l}\vec{y} \\ \vec{z}\end{array}\right]\right\rangle_{\mathcal{H}}=$ $k_{2}\left|z_{3}(L)\right|^{2}$. The compactness follows from the fact that $M^{-1}$ is a canonical isomorphism from $\mathrm{H}$ to $\mathrm{H}^{\prime}$, and the fact that $B$ is a rank-one operator, hence compact from $\mathrm{H}$ to $\mathrm{H}^{\prime}$.

\section{A. Description of $\operatorname{Dom}(\mathcal{A})$}

Proposition 4.2: Let $\vec{u}=(\vec{y}, \vec{z})^{\mathrm{T}} \in \mathcal{H}$. Then $\vec{u} \in$ $\operatorname{Dom}(\mathcal{A})$ if and only if the following conditions hold: $\vec{y} \in\left(H^{2}(0, L) \cap H_{L}^{1}(0, L)\right)^{2} \times\left(H^{3}(0, L) \cap H_{L}^{2}(0, L)\right), \vec{z} \in$ $V$ such that $\left(y_{1}\right)_{x}=\left(y_{2}\right)_{x}=\left.\left(y_{4}\right)_{x x}\right|_{x=L}=0$. Moreover, the resolvent of $\mathcal{A}$ is compact in the energy space $\mathcal{H}$.

Proof: Let $\overrightarrow{\vec{u}}=\left(\begin{array}{c}\overrightarrow{\tilde{y}} \\ \overrightarrow{\tilde{z}}\end{array}\right) \in \mathcal{H}$ and $\vec{u}=\left(\begin{array}{c}\vec{y} \\ \vec{z}\end{array}\right) \in \operatorname{Dom}(\mathcal{A})$ such that $\mathcal{A} \vec{u}=\overrightarrow{\vec{u}}$. Then we have $-\vec{z}=\overrightarrow{\vec{y}} \in V, \quad A \vec{y}+$ $D_{0} z=M \overrightarrow{\tilde{z}}$, and therefore,

$$
\langle\vec{y}, \vec{\varphi}\rangle_{V}=\langle\overrightarrow{\tilde{z}}, \vec{\varphi}\rangle_{H} \text { for all } \vec{\varphi} \in V .
$$

Let $\vec{\psi}=\left[\psi_{1}, \psi_{2}, \psi_{3}\right]^{\mathrm{T}} \in\left(C_{0}^{\infty}(0, L)\right)^{4}$. We define $\varphi_{i}=\psi_{i}$ for $i=1,2$, and $\varphi_{3}=\int_{0}^{x} \psi_{3}(s) d s$. Since $\vec{\varphi} \in V$, inserting $\vec{\varphi}$ into the above equation yields

$$
\begin{aligned}
& \int_{0}^{L}\left\{-\alpha_{1}^{1} h_{1}\left(y_{1}\right)_{x x} \bar{\psi}_{1}-\alpha_{1}^{3} h_{1}\left(y_{2}\right)_{x x} \bar{\psi}_{2}-K_{2}\left(y_{3}\right)_{x x x} \bar{\psi}_{3}\right. \\
& \left.+\frac{G_{2}}{h_{2}}\left(-y_{1}+y_{2}+H\left(y_{3}\right)_{x}\right)\left(-\bar{\psi}_{1}+\bar{\psi}_{2}+H\left(\bar{\psi}_{3}\right)_{x}\right)\right\} d x \\
& +s_{1}\left(z_{1}\right)_{x}(L)\left(\psi_{1}\right)_{x}(L)+s_{3} z_{2}(L) \psi_{2}(L) \\
& +k_{1}\left(z_{3}\right)_{x}(L) \psi_{3}(L)=\int_{0}^{L}\left\{\left(\int_{1}^{x} m \tilde{z}_{4} d s+K_{1}\left(\tilde{z}_{4}\right)_{x}\right) \bar{\psi}_{4}\right. \\
& \left.+\rho_{1} h_{1} \tilde{z}_{1} \bar{\psi}_{1}+\rho_{3} h_{3} \tilde{z}_{2} \bar{\psi}_{2}+\mu h_{3} \tilde{z}_{3} \bar{\psi}_{3}\right\} d x
\end{aligned}
$$

for all $\vec{\psi} \in\left(C_{0}^{\infty}(0, L)\right)^{3}$. Therefore it follows that $\vec{y} \in$ $\left(H^{2}(0, L) \cap H_{L}^{1}(0, L)\right)^{2} \times\left(H^{3}(0, L) \cap H_{L}^{2}(0, L)\right)$.

Next let $\vec{\psi} \in \mathrm{H}$. We define

$$
\varphi_{i}=\int_{0}^{x} \psi_{i}(s) d s, \quad i=1, \ldots, 3 .
$$

Obviously $\vec{\varphi} \in \mathrm{V}$. Then plugging 22 into 21) yields

$$
\begin{aligned}
& 0=\left(\alpha_{1}^{1} h_{1}\left(y_{1}\right)_{x}(1)+s_{1} z_{1}(L)\right) \bar{\psi}_{1}(1)+\alpha_{1}^{3} h_{3}\left(y_{2}\right)_{x}(1) \\
& +s_{3} z_{2}(L) \bar{\psi}_{2}(1)+\left(k_{1}\left(y_{3}\right)_{x x}(L)+k_{1}\left(z_{3}\right)_{x}(L)\right)\left(\bar{\psi}_{3}\right)_{x}(L)
\end{aligned}
$$

for all $\psi \in \mathrm{H}$. Hence,

$$
\begin{aligned}
& \alpha_{1}^{1} h_{1}\left(y_{1}\right)_{x}(1)+s_{1} z_{1}(L)=\alpha_{1}^{3} h_{3}\left(y_{2}\right)_{x}(1)+s_{3} z_{2}(L)=0, \\
& k_{1}\left(y_{3}\right)_{x x}(L)+k_{1}\left(z_{3}\right)_{x}(L)=0 .
\end{aligned}
$$

Now let $\vec{y}=\left[\begin{array}{l}\vec{y}_{1} \\ \vec{y}_{2}\end{array}\right] \in \operatorname{Dom}(\mathcal{A})$ and $\vec{z}=\left[\begin{array}{l}\vec{z}_{1} \\ \vec{z}_{2}\end{array}\right]$ such that $(I+\mathcal{A}) \vec{y}=\vec{z}$. By Proposition 4.2 and Lemma 4.1 . the compactness of the resolvent follows.
Lemma 4.2: The eigenvalue problem

$$
\left\{\begin{array}{l}
\alpha_{1}^{i} h_{i} z_{x x}^{i}-\kappa(i) G_{2} \phi^{2}=\lambda^{2} \rho_{i} h_{i} z^{i}, \quad i=1,3 \\
-K_{2} u_{x x x x}+G_{2} H \phi_{x}^{2}=\lambda^{2}\left(m u-K_{1} u_{x x}\right),
\end{array}\right.
$$

with the overdetermined boundary conditions

$$
\begin{aligned}
& u(0)=u_{x}(0)=z^{i}(0)=z^{i}(L)=z_{x}^{i}(L)=0, \quad i=1,3, \\
& u(L)=u_{x}(L)=u_{x x}(L)=u_{x x x}(L)=0
\end{aligned}
$$

has only the trivial solution.

Proof: Now multiply the equations in 23 by $x \bar{u}_{x}-3 \bar{u}$, $x \bar{z}_{x}^{1}-2 \bar{z}^{1}$, and $x \bar{z}_{x}^{3}-2 \bar{z}^{3}$, respectively, integrate by parts on $(0, L)$, and add them up:

$$
\begin{aligned}
& \int_{0}^{L}\left\{-\alpha_{1}^{1} h_{1}\left|z_{x}^{1}\right|^{2}-\alpha_{1}^{3} h_{3}\left|z_{x}^{3}\right|^{2}-3 \rho_{1} h_{1} \lambda^{2}\left|z^{1}\right|^{2}\right. \\
& -3 \rho_{3} h_{3} \lambda^{2}\left|z^{3}\right|^{2}-4 m \lambda^{2}|u|^{2}-2 K_{1} \lambda^{2}\left|u_{x}\right|^{2} \\
& -G_{2} h_{2} \bar{\phi}^{2}\left(z \phi_{x}^{2}\right)-3 G_{2} h_{2}\left|\phi^{2}\right|^{2}-K_{2} \bar{u}_{x x x x}\left(x u_{x}\right) \\
& +\alpha_{1}^{1} h_{1} \bar{z}_{x x}^{1}\left(x z_{x}^{1}\right)+\alpha_{1}^{3} h_{3} \bar{z}_{x x}^{3}\left(x z_{x}^{3}\right)-\rho_{1} h_{1} \lambda^{2} \bar{z}^{1}\left(x z_{x}^{1}\right) \\
& \left.-\rho_{3} h_{3} \lambda^{2} \bar{z}^{3}\left(x z_{x}^{3}\right)-\lambda^{2}\left(m \bar{u}-K_{1} \bar{u}_{x x}\right)\left(x u_{x}\right)\right) d x=0 .
\end{aligned}
$$

Now consider the conjugate eigenvalue problem corresponding to (23)-(24). Now multiply the equations in the conjugate problem by $x u_{x}+2 u, x z_{x}^{1}+3 z^{1}$, and $x z_{x}^{3}+3 z^{3}$, respectively, integrate by parts on $(0, L)$, and add them up:

$$
\begin{aligned}
& \int_{0}^{L}\left\{3\left(\alpha_{1}^{1} h_{1}\left|z_{x}^{1}\right|^{2}+\alpha_{1}^{3} h_{3}\left|z_{x}^{3}\right|^{2}\right)+3 \bar{\lambda}^{2}\left(\rho_{1} h_{1}\left|z^{1}\right|^{2}\right.\right. \\
& \left.+\rho_{3} h_{3}\left|z^{3}\right|^{2}\right)+2 \bar{\lambda}^{2}\left(m|u|^{2}+K_{1}\left|u_{x}\right|^{2}\right)+2 K_{2}\left|u_{x x}\right|^{2} \\
& +G_{2} h_{2} \bar{\phi}^{2}\left(x \phi_{x}^{2}\right)+3 G_{2} h_{2}\left|\phi^{2}\right|^{2}+K_{2} \bar{u}_{x x x x}\left(x u_{x}\right) \\
& -\alpha_{1}^{1} h_{1} \bar{z}_{x x}^{1}\left(x z_{x}^{1}\right)-\alpha_{1}^{3} h_{3} \bar{z}_{x x}^{3}\left(x z_{x}^{3}\right)+\rho_{1} h_{1} \bar{\lambda}^{2} \bar{z}^{1}\left(x z_{x}^{1}\right) \\
& \left.+\rho_{3} h_{3} \bar{\lambda}^{2} \bar{z}^{3}\left(x z_{x}^{3}\right)+\bar{\lambda}^{2}\left(m \bar{u}-K_{1} \bar{u}_{x x}\right)\left(x u_{x}\right)\right) d x=0 .
\end{aligned}
$$

Finally, adding (25) and (26), considering only the real part of the expression above and all eigenvalues are located on the imaginary axis, i.e. $\lambda=\mp \imath \nu$, yields

$$
\int_{0}^{L}\left(K_{2}\left|u_{x x}\right|^{2}+m \nu^{2}|u|^{2}+\sum_{i=1,3}\left(\alpha_{1}^{i} h_{i}\left|z_{x}^{i}\right|^{2}\right)\right) d x=0 .
$$

This implies that $u=z^{1}=z^{3} \equiv 0$ by 24 . In the case of $\lambda=0$, we have

$$
\left\{\begin{array}{l}
-\phi_{x x}^{2}+\left(\frac{1}{\alpha_{1}^{1} h_{1}}+\frac{1}{\alpha_{1}^{3} h_{3}}\right) \phi^{2}=-H u_{x x x} \\
-K_{2} u_{x x x x}+G_{2} H \phi_{x}^{2}=0,
\end{array}\right.
$$

Since the operator $J=-D_{x}^{2}+\left(\frac{1}{\alpha_{1}^{1} h_{1}}+\frac{1}{\alpha_{1}^{3} h_{3}}\right) I$ is a nonnegative operator on $H_{*}^{2}(0, L)=\left\{\psi \in H^{2}(0, L): \psi(0)=\right.$ $\left.\psi_{x}(L)=0\right\}$, we obtain $-K_{2} u_{x x x x}-G_{2} H^{2}\left(J^{-1} u_{x x x}\right)_{x}=0$, and since $K_{2} D_{x}^{4}+D_{x} J^{-1} D_{x}^{3}$ is a positive operator on its domain, $u=\phi^{2}=0$. And therefore, $u=z^{1}=z^{3} \equiv 0$.

Theorem 4.1: The semigroup generated by $(\mathcal{A}+B)$ is strongly stable in $\mathcal{H}$.

Proof: We know that the system is dissipative, i.e. $\left\langle(\mathcal{A}+B)\left[\begin{array}{c}\vec{z} \\ \dot{\vec{z}}\end{array}\right],\left[\begin{array}{c}\vec{z} \\ \dot{\vec{z}}\end{array}\right]\right\rangle_{\mathrm{H}} \leq 0$. This result together with Lemma 4.2 imply that there are no eigenvalues on the imaginary axis. The conclusion follows.

Remark 4.1: The method used to prove Lemma 4.2 is not valid once we remove the boundary condition $u(L)=0$. An analogous result obtained in [15] was for either clamped, 
hinged, or mixed boundary conditions. It does require $u(L)=0$. The interesting question is whether Theorem 4.1 without $u(L)=0$ is obtained for the case $k_{1} \neq 0, k_{2} \equiv 0$.

Now we consider the decomposition $\mathcal{A}+B=\left(\mathcal{A}_{d}+B\right)+$ $\mathcal{A}_{\phi}$ of the semigroup generator of the original problem 16 where $\mathcal{A}_{d}+B$ is the semigroup generator of the decoupled system, i.e. $\phi^{2} \equiv 0$ in $10-11$,

$$
\left\{\begin{array}{l}
\rho_{i} h_{i} \ddot{v}^{i}-\alpha_{1}^{i} h_{i} v_{x x}^{i}=0, \quad i=1,3, \\
m \ddot{w}-K_{1} \ddot{w}_{x x}+K_{2} w_{x x x x}=0,
\end{array}\right.
$$

with the boundary and initial conditions

$$
\begin{gathered}
v^{i}(0)=w(0)=w_{x}(0)=0, K_{2} w_{x x}(L)=-k_{1} \dot{w}_{x}(L) \\
\alpha_{1}^{i} h_{i} v_{x}^{i}(L)=-s_{i} \gamma^{i} \dot{v}^{i}(L), \quad i=1,3, \\
K_{1} \ddot{w}_{x}(L)-K_{2} w_{x x x}(L)=k_{2} \dot{w}(L) .
\end{gathered}
$$

The operator $\mathcal{A}_{\phi}: \mathcal{H} \rightarrow \mathcal{H}$ is the coupling between the layers defined as the following

$$
\mathcal{A}_{\phi} \mathbf{y}=\left(\begin{array}{c}
0_{3 \times 1} \\
\mathcal{M}^{-1}\left(H G_{2} \phi_{x}^{2}\right) \\
G_{2} \phi^{2} /\left(h_{1} \rho_{1}\right) \\
-G_{2} \phi^{2} /\left(h_{3} \rho_{3}\right)
\end{array}\right)
$$

where $\mathbf{y}=\left(w, u^{1}, u^{3}, \tilde{w}, \tilde{v}^{1}, \tilde{v}^{3}\right) \quad$ and $\quad \phi^{2}=$ $\frac{1}{h_{2}}\left(-u^{1}+u^{3}+H u_{x}\right)$. Let $E_{d}(t)$ be natural energy corresponding to the system $28-22$, i.e. $\phi^{2} \equiv 0$ in 12 .

Theorem 4.2: Let $\mathcal{A}_{d}+B$ be the infinitesimal generator of the semigroup corresponding to the solutions of (28)111. Then the semigroup $\left\{e^{\left(\mathcal{A}_{d}+B\right) t}\right\}_{t \geq 0}$ is exponentially stable in $\mathcal{H}$.

Proof: The exponential stability of the semigroup $e^{\left(\mathcal{A}_{d}+B\right) t}$ follows from the exponential stability of wave equations [15] and the Rayleigh beam equation [17.

Lemma 4.3: The operator $A_{\phi}: \mathcal{H} \rightarrow \mathcal{H}$ defined in 30 is compact.

When $\left(w, u^{1}, u^{3}, \tilde{w}, \tilde{u}^{1}, \tilde{u}^{3}\right) \in \mathrm{H}$, we have $w \in H_{L}^{2}(0, L)$ and $u^{1}, u^{3} \in\left(H_{L}^{1}(0, L)\right)^{2}$, and therefore $\phi^{2} \in H_{L}^{1}(0, L)$. Since $\mathcal{M}: H_{L}^{2}(0, L) \rightarrow \mathrm{L}^{2}(0, L)$ remains an isomorphism, the last terms in 30 satisfy $\mathcal{M}^{-1}\left(\phi_{x}^{2}\right) \in H_{L}^{2}(0, L)$ where $\phi^{2} \in H_{L}^{1}(0, L)$, and $H_{L}^{2}(0, L) \times H_{L}^{1}(0, L)$ is compactly embedded in $H_{L}^{1}(0, L) \times\left(\mathrm{L}^{2}(0, L)\right)^{2}$. Hence the operator $A_{\phi}$ is compact in $\mathcal{H}$.

Theorem 4.3: Then the semigroup $\left\{e^{(\mathcal{A}+B) t}\right\}_{t \geq 0}$ is exponentially stable in $\mathcal{H}$.

Proof: The semigroup $\mathcal{A}+B=\mathcal{A}_{d}+B+\mathcal{A}_{\phi}$ is strongly stable on $\mathcal{H}$ by Theorem 4.1 and the operator $\mathcal{A}_{\phi}$ is a compact in $\mathcal{H}$ by Lemma 4.3. Therefore, since the semigroup generated by $\left(\mathcal{A}_{d}+B+\mathcal{A}_{\phi}\right)-\mathcal{A}_{\phi}$ is uniformly exponentially stable in $\mathcal{H}$ then the semigroup $\mathcal{A}=\left(\mathcal{A}_{d}+B+\mathcal{A}_{\phi}\right)$ is uniformly exponentially stable in $\mathcal{H}$ by e.g., the perturbation theorem of [21.

\section{Future RESEARCH}

A relevant research problem under investigation is whether we can recover the polynomial stability for certain combinations of material properties and for more regular initial data for the composite (8)-(9). For a single piezoelectric beam, this question is answered in [12].

The stabilization results obtained in this paper can be compared to the ones corresponding to the charge or current actuation ([11], [14]).

\section{REFERENCES}

[1] A. Baz, Boundary Control of Beams Using Active Constrained Layer Damping, J. Vib. Acoust. 119-2 (1997), 166-172.

[2] C.Y.K. Chee, L. Tong, and G.P. Steven, A review on the modelling of piezoelectric sensors and actuators incorporated in intelligent structures, J. Intel. Mater. Syst. Str. 9 (1998), 3-19.

[3] R.A. DiTaranto, Theory of vibratory bending for elastic and viscoelastic layered finitelength beams, J. Appl. Mech. 32 (1965), 881-886.

[4] S.W. Hansen, Several Related Models for Multilayer Sandwich Plates, Math. Models \& Methods in Applied Sciences 14-8 (2004), 1103-1132.

[5] S.W. Hansen, A.Ö. Özer, Exact boundary controllability of an abstract Mead-Marcus Sandwich beam model, The Proceedings of $49^{\text {th }}$ IEEE Conf. on Decision \& Control, Atlanta, USA (2010), 2578-2583.

[6] J.E. Lagnese, J.-L. Lions, Modeling Analysis and Control of Thin Plates, (Masson, Paris, 1988).

[7] M.J. Lam, D. Inman, W. R. Saunders, Vibration Control through Passive Constrained Layer Damping and Active Control, J. Intel. Mater. Syst. Str. 8-8 (1997), pp. 663-677.

[8] D.J. Mead and S. Markus, The forced vibration of a three-layer, damped sandwich beam with arbitrary boundary conditions, $J$. Sound Vibr. 10 (1969), 163-175.

[9] S. Miller and J.Jr. Hubbard, Observability of a Bernoulli Euler Beam using $\mathrm{PVF}_{2}$ as a Distributive Sensor, The Seventh Conference on Dynamics \& Control of Large Structures, VPI \& SU, Blacksburg, VA (1987), 375-390.

[10] K.A. Morris, A.Ö. Özer, Modeling and stabilizability of voltageactuated piezoelectric beams with magnetic effects, SIAM J. Control Optim. 52-4 (2014), 2371-2398.

[11] K.A. Morris, A.Ö. Özer, Comparison of stabilization of currentactuated and voltage-actuated piezoelectric beams, the $53^{\text {rd }}$ Proceedings of the IEEE Conf. on Decision \& Control, Los Angeles, California, USA (2014), 571-576.

[12] A.Ö. Özer, Further stabilization and exact observability results for voltage-actuated piezoelectric beams with magnetic effects, Math. of Control, Signals, and Systems 27-2 (2015), 219-244.

[13] A.Ö. Özer, Semigroup well-posedness of a voltage controlled active constrained layered (ACL) beam with magnetic effects, The Proceedings of the American Control Conference, Boston, MA, USA (2016), 4580-4585.

[14] A.Ö. Özer, Potential formulation and related stabilization results for a charge or current-controlled piezoelectric smart composite: electrostatic, quasi-static, and fully-dynamic assumptions, submitted.

[15] A.Ö. Özer, and S.W. Hansen, Uniform stabilization of a multilayer Rao-Nakra sandwich beam, Evolution Equations and Control Theory 2-4 (2013), 195-210.

[16] Y.V.K.S. Rao and B.C. Nakra, Vibrations of unsymmetrical sandwich beams and plates with viscoelastic cores, J. Sound Vibr. 34-3 (1974), 309-326.

[17] B. Rao, A compact perturbation method for the boundary stabilization of the Ragleigh beam equation, Appl. Math. Optim., 3-33, pp. 253-264, 1996.

[18] I.Y. Shen, A variational formulation, a work-energy relation and damping mechanisms of active constrained layer treatments, Journal of Vibration and Acoustics 119-2 (1997), 192-199.

[19] R.C. Smith, Smart Material Systems, (Society for Industrial and Applied Mathematics, 2005).

[20] R. Stanway, J.A. Rongong, N.D. Sims, Active constrainedlayer damping: A state-of-the-art review, Automation \& Control Systems 217-6 (2003), 437-456.

[21] R. Triggiani, Lack of uniform stabilization for noncontractive semigroups under compact perturbation, Proc. Amer. Math. Soc. (105) (1989), 375-383. 
[22] M. Trindade and A. Benjendou, Hybrid Active-Passive Damping Treatments Using Viscoelastic and Piezoelectric Materials:Review and Assessment, J. Vib. and Cont. 8-6 (2002), 699745 . 